\title{
Deficits in Col5a2 Expression Result in Novel Skin and Adipose Abnormalities and Predisposition to Aortic Aneurysms and Dissections
}

Arick C. Park, ${ }^{*}$ Noel Phan, ${ }^{\dagger}$ Dawiyat Massoudi, ${ }^{*}$ Zhenjie Liu, ${ }^{\dagger}$ John F. Kernien, ${ }^{\star}$ Sheila M. Adams, ${ }^{\ddagger}$ Jeffrey M. Davidson, David E. Birk, ${ }^{\ddagger}$ Bo Liu, ${ }^{\dagger}$ and Daniel S. Greenspan*

From the Departments of Cell and Regenerative Biology* and Surgery, ${ }^{\dagger}$ University of Wisconsin, Madison, Wisconsin; the Department of Molecular Pharmacology and Physiology, ${ }^{\ddagger}$ Morsani College of Medicine, University of South Florida, Tampa, Florida; and the Department of Pathology, Microbiology, and Immunology, ${ }^{\S}$ Vanderbilt University, Nashville, Tennessee

\author{
Accepted for publication \\ June 8, 2017. \\ Address correspondence to \\ Daniel S. Greenspan, Ph.D., \\ Department of Cell and Regen- \\ erative Biology, School of \\ Medicine and Public Health, \\ University of Wisconsin, 1111 \\ Highland Ave, Madison, \\ WI 53705. E-mail: dsgreens@ \\ wisc.edu.
}

\begin{abstract}
Classic Ehlers-Danlos syndrome (cEDS) is characterized by fragile, hyperextensible skin and hypermobile joints. cEDS can be caused by heterozygosity for missense mutations in genes COL5A2 and COL5A1, which encode the $\alpha 2(\mathrm{~V})$ and $\alpha 1(\mathrm{~V})$ chains, respectively, of collagen $\mathrm{V}$, and is most often caused by COL5A1 null alleles. However, COL5A2 null alleles have yet to be associated with CEDS or other human pathologies. We previously showed that mice homozygous null for the $\alpha 2(\mathrm{~V})$ gene Col5a2 are early embryonic lethal, whereas haploinsufficiency caused aberrancies of adult skin, but not a frank cEDS-like phenotype, as skin hyperextensibility at low strain and dermal cauliflower-contoured collagen fibril aggregates, two cEDS hallmarks, were absent. Herein, we show that ubiquitous postnatal Col5a2 knockdown results in pathognomonic dermal cauliflower-contoured collagen fibril aggregates, but absence of skin hyperextensibility, demonstrating these cEDS hallmarks to arise separately from loss of collagen $\mathrm{V}$ roles in control of collagen fibril growth and nucleation events, respectively. Col5a2 knockdown also led to loss of dermal white adipose tissue (WAT) and markedly decreased abdominal WAT that was characterized by miniadipocytes and increased collagen deposition, suggesting $\alpha 2(\mathrm{~V})$ to be important to WAT development/maintenance. More important, Col5a2 haploinsufficiency markedly increased the incidence and severity of abdominal aortic aneurysms, and caused aortic arch ruptures and dissections, indicating that $\alpha 2(\mathrm{~V})$ chain deficits may play roles in these pathologies in humans. (Am J Pathol 2017, 187: 2300-2311; http://dx.doi.org/10.1016/j.ajpath.2017.06.006)
\end{abstract}

Type $\mathrm{V}$ collagen $[\operatorname{col}(\mathrm{V})]$ is a quantitatively minor fibrilforming collagen broadly expressed in vertebrate tissues as an $\alpha 1(\mathrm{~V})_{2} \alpha 2(\mathrm{~V})$ heterotrimer, comprising one $\alpha 2(\mathrm{~V})$ and two $\alpha 1(\mathrm{~V})$ chains. $^{1}$ In addition, $\alpha 1(\mathrm{~V})_{3}$ homotrimers and $\alpha 1(\mathrm{~V}) \alpha 2(\mathrm{~V}) \alpha 3(\mathrm{~V})$ heterotrimeric forms of $\operatorname{col}(\mathrm{V})$ also have been reported in tissues, although the significance and functions of such forms are only beginning to be explored. $^{2-6}$ Early evidence suggested a pericellular or basement membrane-associated distribution for $\operatorname{col}(\mathrm{V})$ forms. ${ }^{7,8}$ However, subsequent studies have demonstrated that $\alpha 1(\mathrm{~V})_{2} \alpha 2(\mathrm{~V})$ heterotrimers are incorporated into the growing fibrils of the much more abundant fibrillar collagen I fibrils, and play roles in regulating the nucleation, size, and shapes of the resulting collagen $\mathrm{I} / \operatorname{col}(\mathrm{V})$ heterotypic fibrils. ${ }^{9-11}$

Human patients with the heritable connective tissue disorder classic Ehlers-Danlos syndrome (cEDS) present with skin hyperextensibility, joint hypermobility, atrophic scarring, and easy bruising. ${ }^{12}$ Most cEDS cases are due to heterozygosity for null alleles for the $\alpha 1(\mathrm{~V})$ gene

\footnotetext{
Supported by the Department of Veterans Affairs (J.M.D.), the University of Wisconsin School of Medicine and Public Health (D.S.G.), and NIH grants AR044745 (D.E.B.), R01HL088447 (B.L.), and AR047746 and AI084853 (D.S.G.).

Disclosures: None declared.
} 
COL5A1. ${ }^{12,13}$ Similarly, a null allele in the mouse $\alpha 1(\mathrm{~V})$ gene Col5al causes a cEDS-like phenotype in mice. ${ }^{14,15}$ In addition, a number of human cEDS cases have now been shown to be due to heterozygosity for missense mutations in COL5A1, or in the $\alpha 2(\mathrm{~V})$ gene COL5A2. ${ }^{12}$ Interestingly, however, no human cEDS case has yet been associated with heterozygosity for a COL5A2 null allele, leading to the suggestion that haploinsufficiency for the $\alpha 2(\mathrm{~V})$ chain may not lead to cEDS or, perhaps, to any clinically abnormal phenotype. $^{12}$

To determine in vivo effects of reduced or absent $\alpha 2(\mathrm{~V})$ chains, we recently generated and characterized mice with constitutively null Col5a2 alleles. ${ }^{16}$ Homozygosity for the null Col5a2 allele yielded embryonic lethality at approximately 12 days after conception, ${ }^{16}$ approximately 2 days later than the embryonic lethality previously described for homozygous null Colsal ${ }^{-1-}$ mice, ${ }^{11}$ although embryos of both genotypes had similar cardiovascular signs, suggesting deficits in early cardiovascular integrity. However, although $\mathrm{Col} \mathrm{al}^{-1-}$ embryos lacked identifiable mesenchymal collagen fibrils, ${ }^{11}$ Col5a2 $2^{-1-}$ embryos had identifiable mesenchymal collagen fibrils, albeit with abnormally large diameters and abnormal configurations. ${ }^{16}$ Differences in collagen fibrils and survival time between the two types of homozygous null embryos suggested that aberrant $\alpha 1(\mathrm{~V})_{3}$ homotrimers, which are formed by $\alpha 1(\mathrm{~V})$ chains in the absence of $\alpha 2(\mathrm{~V})$ chains, ${ }^{17,18}$ can functionally compensate, in part, for loss of $\alpha 1(\mathrm{~V})_{2} \alpha 2(\mathrm{~V})$ heterotrimers.

Unlike heterozygous $\mathrm{Colsal}^{+/-}$adult mice, which exhibit hyperextensible skin at both high and low stress, and on electron microscopic examination display large-diameter dermal aggregates of collagen fibrils with cauliflower-like contours ${ }^{14}$ cardinal features of cEDS, ${ }^{12}$ adult heterozygous Colsa $2^{+/-}$mice exhibited hyperextensible skin at high, but not low, stress and dermal collagen fibrils with relatively mild abnormalities in contour and diameter. ${ }^{16}$ Together, these data suggested that human $C O L 5 A 2^{+/-}$heterozygotes, although likely more susceptible to skin tearing/wounding than normal, might not present with frank cEDS. This conclusion, together with the finding that aortas of $\mathrm{Col}_{5 \mathrm{a}}{ }^{+/-}$heterozygous mice have reduced tensile strength and increased elasticity, raised the question of the clinical ramifications of the COL $5 A 2^{+/-}$genotype in human populations. ${ }^{16}$

In the current report, we further elucidated the roles of $\alpha 2(\mathrm{~V})$ collagen chain in adult tissues and pathology, in part by using a conditional-null mouse model with floxed Cols 2 alleles that avoids embryonic lethality and allows study of the effects of postnatal Col5a2 ablation. Data presented herein show the $\alpha 2(\mathrm{~V})$ chain to play important roles in growth and that sufficiently low $\alpha 2(\mathrm{~V})$ chain levels yield skin that, like that of cEDS patients, ${ }^{19}$ has delayed and aberrant wound healing, has greatly reduced breaking strength/tensile strength, and contains the large cauliflower-like collagen fibril aggregates pathognomic of cEDS. ${ }^{16}$ However, unlike skin of cEDS patients, this skin lacks hyperextensibility and had almost total absence of dermal white adipose tissue
(WAT). The latter, along with abdominal WAT depots of reduced size and fibrotic appearance, and in which adipocytes had a miniadipocyte appearance, suggests a role for $\alpha 2(\mathrm{~V})$ in WAT development and/or maintenance. Key observations include the finding that $\alpha 2(\mathrm{~V})$ chain haploinsufficiency yields markedly increased incidence, diameters, and pathology of abdominal aortic aneurysms (AAAs), and high susceptibility to fatal aortic arch ruptures and dissections in an angiotensin II-induced aneurysm mouse model. Thus, deficits in $\alpha 2(\mathrm{~V})$ chain levels/function may play roles in these pathologies in humans.

\section{Materials and Methods}

\section{Col5a2-Floxed Mice}

The generation of $\mathrm{Col} 5 \mathrm{a} 2^{f l / f}$ mice has been described previously. ${ }^{16}$

\section{Induced Deletion of Col5a2}

C57BL/6 Col5a $2^{A l / f}$ mice were mated with transgenic CreERT2 mice in which Cre recombinase expression was driven by the ubiquitin $\mathrm{C}$ (Ubc) promoter (JAX strain 008085), ${ }^{20}$ to generate $U b c$ Cre-ERT2; Colsa $2^{f / f l}$ mice. Excision of Col5a2 sequences was then induced with tamoxifen, administered into the peritoneum (i.p.) of recently weaned 4 -week-old male mice $(0.5 \mu \mathrm{mol} / \mathrm{L}$ per gram body weight), weighing approximately 13 to $15 \mathrm{~g}$, for a total of 10 days (two blocks of 5 consecutive days separated by 2 days without treatment). Littermates without the $U b c$ Cre-ERT2 transgene were used as controls and were similarly treated with tamoxifen. Efficacy of tamoxifeninduced excision of Colsa2 was assayed via PCR analysis of genomic DNA from ear samples digested in DirectPCR Lysis Reagent (Viagen Biotech Inc., Los Angeles, CA). Oligonucleotide forward (5'-GGTGATGGATGCTGACTTTG-3' $/ 5^{\prime}$-AGCTTCTGTGCGTGCCCTGG-3') and reverse (5'-GGAGGGGAGGATAAAGAGCA- $\left.3^{\prime}\right)$ primers were used to detect the presence of floxed or excised alleles, represented by approximately 460- and approximately 510bp bands, respectively, on resolution on $2.5 \%$ agarose gels.

$\mathrm{Col} 5 \mathrm{a} 2^{f / f l}$ mice also were mated with transgenic CreERT2 mice, in which Cre expression is driven by the myosin heavy chain 11 promoter (JAX strain 019079), ${ }^{21}$ specific to smooth muscle cells, to generate Myh11 Cre-ERT2; $\operatorname{Col} a 2^{f / f l}$ mice. Male mice, all of which must contain the Y chromosome-linked Myhl1 Cre-ERT2 transgene, were injected with either tamoxifen or vehicle (98\% corn oil and $2 \%$ ethanol) using the same dosage and administration schedule as described above for $\mathrm{Ubc}$-Cre; $\mathrm{Col} 5 \mathrm{a} 2^{f / f l}$ mice.

All mice were housed and treated in accordance with NIH's Guide for the Care and Use of Laboratory Animals, ${ }^{22}$ using protocols approved by the Research Animal Resources Center of the University of Wisconsin-Madison. 


\section{Splinted Wound Healing}

Splinted wound healing was performed on 8- to 10-week-old mice, as described previously. ${ }^{23}$ Briefly, all wounding procedures were performed under isoflurane anesthesia. The backs of mice were shaved and sterilized. Two full-thickness wounds were generated on the midback using a 5-mm biopsy tool (Integra Miltex, York, PA). Circular, silicon splints, with a 6-mm inner diameter, were adhered around the edges of the wound using cyanoacrylate glue (Elmer's Products, Atlanta, GA) and fastened in place with four interrupted sutures. Wounds were covered by protective dressings for the duration of the experiment. Wound closure was analyzed using calipers by measuring two diameters per wound and using the average to estimate wound area. After sacrifice, the wounded tissue was carefully dissected for further analysis.

\section{Dermal Fibroblast Culturing and Immunoblotting}

Full-thickness skin was excised from the axillary region of 15week-old adult mice. The tissue was then minced using a pair of scalpels and was digested overnight in $0.5 \mathrm{mg} / \mathrm{mL}$ type II collagenase (Worthington Biochemical Corporation, Lakewood, NJ) in Dulbecco's modified Eagle's medium supplemented with $20 \%$ fetal bovine serum, in a $5 \% \mathrm{CO}_{2}$ atmosphere at $37^{\circ} \mathrm{C}$. The digest was pelleted at $1500 \times g$ for 10 minutes, the supernatant was removed, and the pellet was subsequently resuspended and plated in Dulbecco's modified Eagle's medium and $20 \%$ fetal bovine serum growth medium.

For immunoblotting, cells were washed twice with phosphate-buffered saline to remove residual growth medium before being serum starved in Dulbecco's modified Eagle's medium supplemented with $75 \mu \mathrm{g} / \mathrm{mL}$ ascorbic acid and $40 \mu \mathrm{g} / \mathrm{mL}$ soybean trypsin inhibitor (Sigma, St. Louis, MO). The following day, conditioned media were collected and concentrated by centrifugation filters (Millipore, Billerica, MA). Samples were boiled in Laemmli sample buffer and $2.5 \% \quad \beta$-mercaptoethanol before loading onto a $6 \%$ acrylamide gel and SDS-PAGE. Primary antibodies specific for the mouse $\alpha 1(\mathrm{~V})$ proline/arginine-rich protein and variable domains or the $\alpha 2(\mathrm{~V})$ cysteine-rich domain were used, as previously described. ${ }^{16}$ Anti-procollagen C-proteinase enhancer 1 antibody (Sigma) was used at a 1:1000 dilution. Goat anti-rabbit horseradish peroxidase-conjugated secondary antibody (Bio-Rad Laboratories Inc., Hercules, CA) was used at a 1:5000 dilution. Detection was with chemiluminescent substrate (Thermo Scientific, Waltham, MA).

\section{Histologic Analyses}

Hair was removed from subscapular skin samples, and WAT depots were isolated; samples were then fixed overnight in $10 \%$ formalin, followed by embedding in paraffin, and dividing into sections ( $5 \mu \mathrm{m}$ thick). For hematoxylin and eosin staining, sections were fixed for 5 minutes in $10 \%$ formalin, then rinsed with water, and stained in modified
Harris hematoxylin solution (Newcomer Supply, Inc., Middleton, WI) for 4 minutes. Samples were dehydrated in $1 \%$ acid alcohol, and the nuclei were blued in $1 \%$ ammonia water for 30 seconds. Cytoplasmic staining was achieved by immersing the slides in $1 \%$ alcoholic eosin $\mathrm{Y}$ solution (Newcomer Supply, Inc.). Slides were then dehydrated through $95 \%$ and $100 \%$ ethanol, cleared in xylene, and mounted with Cytoseal 60 (Thermo Fisher).

For trichrome staining, sections were fixed for 5 minutes in $10 \%$ formalin and placed in Bouin's solution for 1 hour at $60^{\circ} \mathrm{C}$. Slides were washed until tissue sections were clear. Tissue nuclei were stained with working Wiegert's Iron Hematoxylin solution (Newcomer Supply, Inc.) for $10 \mathrm{mi}-$ nutes. Slides were then stained for collagen with Gomori's one-step trichrome solution (Newcomer Supply, Inc.) for 20 minutes. Last, slides were rinsed with $1 \%$ glacial acetic acid to enhance contrast, dehydrated through $95 \%$ and $100 \%$ ethanol, cleared in xylene, and mounted with Cytoseal 60. Bright-field images were captured using a Zeiss Axiophot 2 microscope (Zeiss, Thornwood, NY) with an attached charge-coupled device camera.

\section{Transmission Electron Microscopy}

Mouse subscapular skin was fixed in $4 \%$ paraformaldehyde and $2.5 \%$ glutaraldehyde in $0.1 \mathrm{~mol} / \mathrm{L}$ sodium cacodylate buffer, $\mathrm{pH} 7.4$, with $8.0 \mathrm{mmol} / \mathrm{L} \mathrm{CaCl}_{2}$ for 2 to 4 hours and post-fixed with $1 \%$ osmium tetroxide. Further sample preparation and imaging was as previously described. ${ }^{16}$

\section{Biomechanical Analysis of Skin}

Tensiometric properties of moist skin strips $(20 \times 30 \mathrm{~mm}$, trimmed to generate a $5-\mathrm{mm}$ waist that provided a uniform breaking pattern) from the dorsal midline were evaluated using an Instron Tensiometer (model 5542; Instron, Canton, MA). Mechanical properties (breaking energy and Young's modulus) were calculated with Bluehill software version 2.32 (Instron, Norwood, MA).

\section{Elastase and Angiotensin II Infusion Models for Aortic Aneurysms}

For the elastase model, 12- to 15-week-old adult mice were anesthetized and underwent sterile preparation. A laparotomy was performed to expose the abdominal aorta. The aorta was temporarily ligated at two ends, and an aortotomy was performed between the ligations. Through the aortotomy, the aorta was perfused with $0.414 \mathrm{U} / \mathrm{mL}$ type I porcine pancreatic elastase (Sigma) in saline for 5 minutes at a pressure of $100 \mathrm{mmHg}$. After perfusion, the aortotomy was closed and the ligations were removed. Preperfusion measurements of aorta diameters were recorded using digital calipers, and measurements were taken again on sacrifice 2 weeks after surgery. The percentage change in diameter was calculated by comparing these two values. 

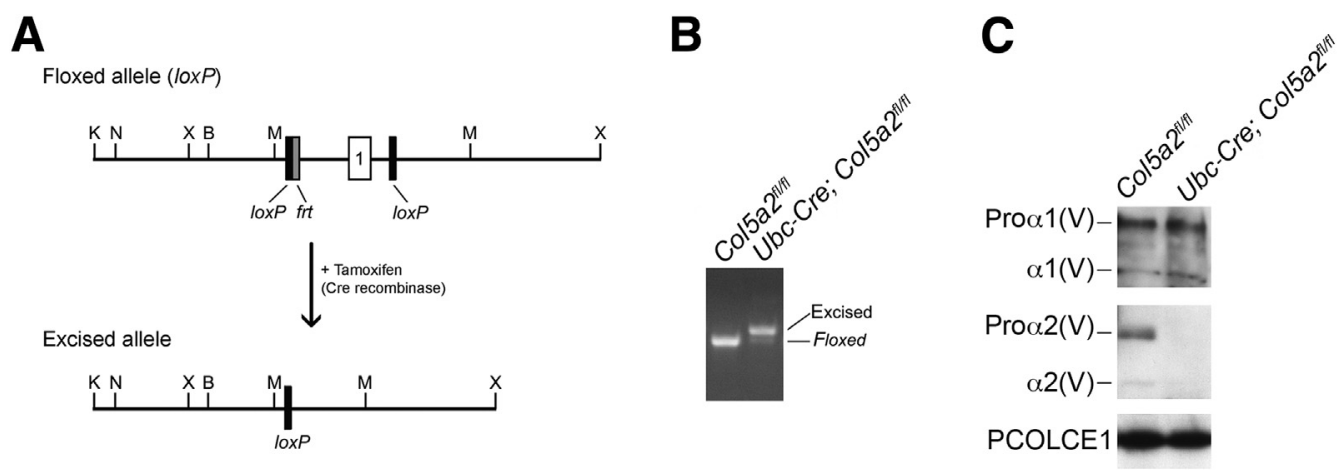

Figure 1 Tamoxifen-induced Col5a2 ablation. A: Schematic shows placement of loxP and frt sequences, represented in black and gray, respectively. LoxP sequences flank exon 1 (open box) and adjoining promoter and enhancer sequences. On tamoxifen treatment, site-specific excision by induced Cre recombinase deletes LoxP-flanked sequences to produce the excised (ablated) allele. B: PCR genotyping of genomic DNA from Col5a $2^{f l f l}$ and $U b c-C r e ; C o l 5 a 2^{f l / f l}$ mice treated with tamoxifen shows genomic excision of exon 1 only in mice $\left(U b c-C r e ; C o l 5 a 2^{f / f l}\right)$ harboring the $U b c-C r e$ transgene. C: Immunoblotting of conditioned media from primary dermal fibroblasts isolated from Ubc-Cre; Col5a $2^{f / f l}$ mice shows a striking deficit in $\alpha 2(\mathrm{~V})$ chain secretion, compared with Col5a $2^{f l / f l}$ fibroblasts. Both types of fibroblasts are seen to secrete similar levels of $\alpha 1(\mathrm{~V})$ chains. Procollagen C-proteinase enhancer 1 (PCOLCE1) was used as a loading control. B, BamH1; K, KpnI; M, MfeI; N, NcoI; X, XbaI.

For the angiotensin II (Ang II) infusion model, constitutively heterozygous null Col5a2 ${ }^{+/-}$mice $^{16}$ were crossed with $\mathrm{Ldlr}^{-1-}$ mice (Jackson Laboratory, Bar Harbor, ME), and $\mathrm{Col}_{5 \mathrm{a2}}{ }^{+/-} ; \mathrm{Ldlr}^{-/-}$and $\mathrm{Col} 5 \mathrm{a2^{+/+ }} ; \mathrm{Ldlr}^{-/-}$control male mice at the age of 6 to 8 months were subjected to a 28-day infusion of Ang II. Alzet model 2004 osmotic minipumps (Durect Corp., Cupertino, CA), implanted s.c., were used to deliver Ang II (1000 ng/kg per minute; SigmaAldrich, St. Louis, MO) or vehicle (normal saline). Mice were anesthetized with isoflurane and administered buprenorphine $(0.05$ to $0.10 \mathrm{mg} / \mathrm{kg})$ via i.p. injection after the procedure. At the completion of Ang II infusion, mice were perfused with normal saline, followed by $4 \%$ paraformaldehyde, at $100 \mathrm{mmHg}$ for 5 minutes. Periadventitial tissues were carefully removed from the aortic wall, and a digital caliper (Absolute Digimatic; Mitutoyo, Aurora, IL) was used to measure maximal external diameters of suprarenal abdominal aortas. For this study, AAA was defined as $\geq 50 \%$ enlargement of maximal abdominal aorta diameter. Characterization of the severity of AAA formation was as previously described ${ }^{24}$ : type I, dilated lumen in the suprarenal aorta with no thrombus; type II, remodeled tissue in the suprarenal region that frequently contains thrombus; type III, a pronounced bulbous form of type II that contains thrombus; and type IV, multiple aneurysms containing thrombus, some overlapping, in the suprarenal area of the aorta. Necropsy was performed as soon as possible on animals that expired before completion of the study. These animals were not included in the histologic or AAA quantification analyses, but only in the mortality data.

\section{Statistical Analysis}

Data are expressed as means \pm SEM. To compare incidence of AAA formation between the two groups, $\chi^{2}$ test and twotailed Fisher's exact probability test were used. A two-tailed $t$-test for normally distributed data and a Mann-Whitney $U$ nonparametric test for skewed data that deviate from normality were used to compare two conditions. Differences with $P<0.05$ were considered significant.

\section{Results}

\section{Validation of Col5a2 Conditional Knockout}

To determine the effects of ablating Col5a2 function in juvenile and adult mice, the embryonic demise associated with constitutive loss of Col5a2 function ${ }^{16}$ was avoided via conditional Col5a2 knockdown. Toward this end, mice were generated in which exon 1 and adjoining promoter and enhancer regions were flanked with flox (loxP) sites. Crossing with transgenic mice in which Cre expression is driven by the ubiquitin $\mathrm{C}$ promoter generated $\mathrm{Ubc}$-Cre; Col5a $2^{f / f l}$ mice, in which Cre activation by administration of tamoxifen excises Col5a2 sequences flanked by the loxP sites (Figure 1A). Genomic DNA extracted from ear punch samples after tamoxifen administration showed an upward mobility shift of approximately $50 \mathrm{bp}$ from $\mathrm{Ubc}$-Cre; Colsa $2^{f / f l}$ DNA, indicating genomic excision by Cre, unlike Colsa $2^{f / f l}$ littermate controls, which were also administered tamoxifen, but lacked the Ubc-Cre transgene (Figure 1B). Moreover, neither secreted pro- $\alpha 2(\mathrm{~V})$ chains nor mature $\alpha 2(\mathrm{~V})$ chains were detected in cultures of dermal fibroblasts isolated from 15-week-old tamoxifen-treated adult $\mathrm{Ubc}$ - $\mathrm{Cre}$; Col5a $2^{f l f l}$ mice (Figure 1C).

\section{Ubc-Cre; Col5a $2^{f l f l}$ Mice Are Runted and Have Abnormalities of Skin and WAT}

Ubc-Cre; $\mathrm{Col} 5 a 2^{f / f t}$ mice failed to gain weight subsequent to tamoxifen treatment and had significantly decreased weight, compared with tamoxifen-treated Col5 $\mathrm{a}^{\mathrm{fl} / \mathrm{f}}$ 

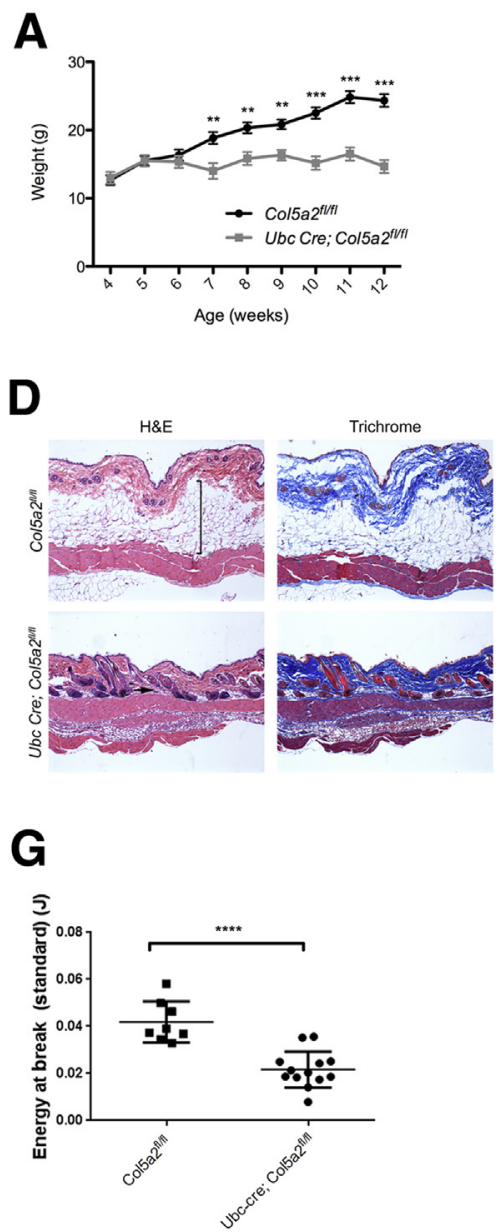
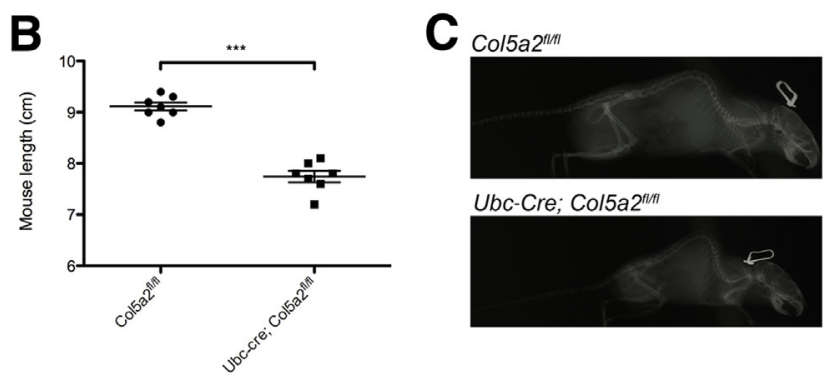

$\mathbf{E}$
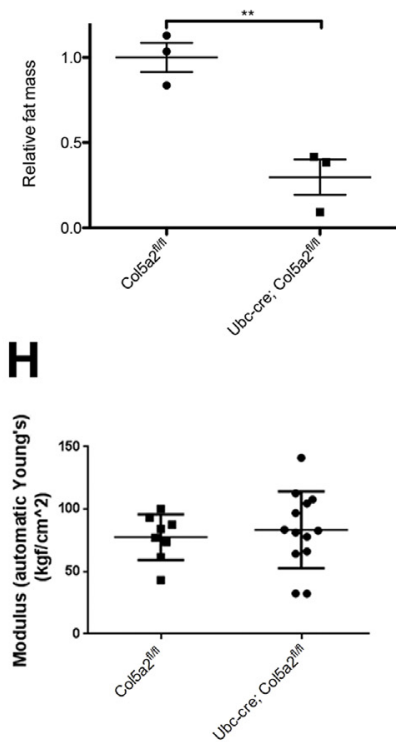

$\mathbf{F}$
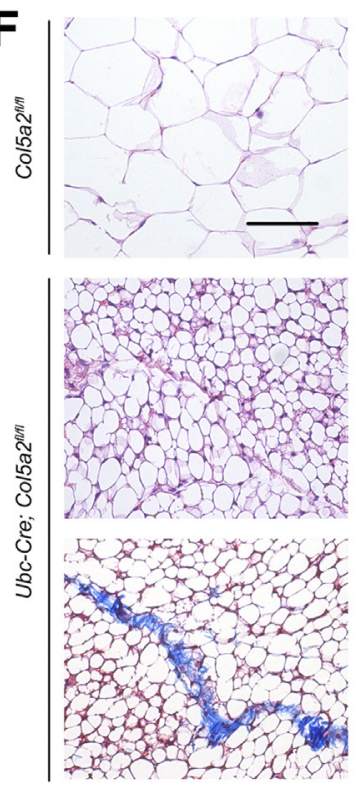

Figure 2 Morphologic appearance and features of skin and white adipose tissue (WAT) depots of Col5a2 adult mutant mice. A-C: Conditional loss of Col5a2 function results in markedly reduced mass (A) and length (B), and severe kypholordosis (C). The white outlined object above the head of each mouse in C is the ear tag. D: Hematoxylin and eosin (H\&E) and Masson's trichrome staining of subscapular skin samples. Vertical bracket and arrow denote dermal WAT in Ubc-Cre; Col5a ${ }^{f l f l}$ and Col5a $2^{f l f l}$ littermate control skin, respectively. E: Masses of inguinal fat depots, normalized to the body weights of the mice from

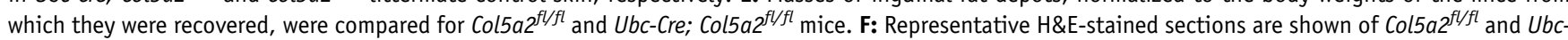
Cre; Col5a $2^{f l f l}$ inguinal fat depots. Masson's trichrome staining of a section of $U b c$-Cre; Col5a ${ }^{f l f l}$ inguinal fat depot is shown, evidencing a characteristic collagenous seam. Although only data for inguinal fat depots are shown in $\mathbf{E}$ and $\mathbf{F}$, results were similar for gonadal fat depots. $\mathbf{G}$ and $\mathbf{H}$ : Biomechanical measurement shows Ubc-Cre; Col5a $2^{f l f l}$ skin to have markedly decreased breaking strength $(P<0.0001 ; \mathbf{G})$, but no significant difference $(P<0.638 ; \mathbf{H})$ in extensibility (Young's modulus), compared with control skin. Data are presented as means \pm SEM (A, B, E, G, and $\mathbf{H})$. ${ }^{* *} P<0.01,{ }^{* *} P<0.001$, and $* * * * P<0.0001$. Scale bar $=50 \mu \mathrm{m}(\mathbf{F})$.

controls, as early as 7 weeks of age (approximately 2 weeks after the final tamoxifen treatment). Weight differences further increased until weights of $\mathrm{Ubc}$-Cre; $\mathrm{Col} 5 \mathrm{a} 2^{\mathrm{fl} / \mathrm{fl}}$ mice were only approximately $60 \%$ those of $C o l 5 a 2^{f / f l}$ controls at adulthood (Figure 2A). Ubc-Cre; Colsa $2^{f l f t}$ mice were also of shorter length than Colsa2 $2^{f / f l}$ controls (Figure 2B) and exhibited severe kypholordosis (Figure 2C).

Skin abnormalities are a hallmark of cEDS. We, therefore, subjected subscapular skin samples of $\mathrm{Ubc-Cre}$; $\mathrm{Col} 5 \mathrm{a} 2^{f / f l}$ and control mice to histologic analysis. Hematoxylin and eosin staining showed Ubc-Cre; Colsa $2^{f / f t}$ dermis to be markedly thinner, and seemingly more compact than control skin (Figure 2D). Masson's trichrome staining of serial sections confirmed a thinner, more compact, dermis and also suggested $\mathrm{Ubc}$-Cre; $\mathrm{Col} 5 \mathrm{a}^{f / / f l}$ dermis to be more collagen dense than control dermis (Figure 2D). Ubc-Cre;
Col5a $2^{f / f}$ skin also apparently lacked dermal WAT, with an absence of morphologically identifiable adipocytes (Figure 2D). Consistent with these histologic results, on handling of mice, a thinning of the skin was readily apparent, and most evident while grasping mice by the scruff of the neck or other areas of dorsal skin.

To determine whether loss of WAT was limited to $U b c$ Cre; Col5a $2^{f / f l}$ skin, inguinal and gonadal fat depots were

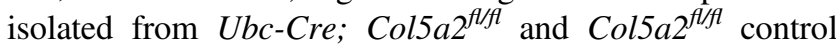
mice and their weights were compared. Although $\mathrm{Ubc-Cre}$; $\mathrm{Col} 5 \mathrm{a} 2^{f / f l}$ mice are approximately $60 \%$ the weight and approximately $60 \%$ the length of $\mathrm{Col} 5 \mathrm{a} 2^{\mathrm{flf} f}$ controls (Figure 2, A and B), Ubc-Cre; Colsa $2^{f / f t}$ inguinal and gonadal fat depots were only $18 \%$ and $15 \%$ the mass of the corresponding $\mathrm{Col} 5 \mathrm{a} 2^{f / f l}$ depots, respectively; and were approximately $30 \%$ and approximately $25 \%$, respectively, 
when normalized to the body weights of the mice from which they were recovered (Figure 2E). As Ubc-Cre; Col5a2 $2^{f / f l}$ mice had food intake approximately $60 \%$ that of Colsa $2^{f l f l}$ mice (data not shown), and thus proportional to their reduced size, the aggregate data from the much reduced WAT of skin and abdominal depots suggest that the $\alpha 2(\mathrm{~V})$ chain plays a role in WAT development and/or maintenance. Consistent with this possibility, the adipocytes in $\mathrm{Ubc}$-Cre; $\mathrm{Col} 5 \mathrm{C2}^{f / f l}$ inguinal and gonadal WAT depots were miniadipocytes, of an abnormally small size (Figure 2F). Ubc-Cre; Col5a $2^{f l / f}$ inguinal and gonadal WAT depots were also abnormal in having seams of dense collagenous matrix not found in control inguinal or gonadal WAT depots (Figure 2F). These fibrotic seams in abdominal WAT depots were reminiscent of the dense collagenous matrix in $\mathrm{Ubc}$-Cre; $\mathrm{Col} 5 \mathrm{a} 2^{f / f l}$ skin (Figure 2D), and are evidence of an abnormal collagenous matrix in both tissues.

\section{Ubc-Cre; Col5a $2^{f l / f l}$ Skin Is Markedly Fragile, But Not Hyperextensible}

To determine whether these differences translated into differences in mechanical strength, skin samples were examined for tensile strength, the amount of force required for the skin to stretch and break. Mechanical testing, and analysis that included correction for the thinner dorsal skin of $\mathrm{Ubc}$-Cre; Col5a $\mathrm{flllf}^{f /}$ skin specimens, showed the latter to have markedly reduced breaking energy (toughness), with the breaking energy of $\mathrm{Ubc}$-Cre; Col5a $2^{f f l}$ skin reduced by $49 \%$ compared with control skin $(P<0.0001)$ (Figure $2 \mathrm{G})$. Surprisingly, however, the elastic modulus of $\mathrm{Ubc-Cre}$; Col5a $2^{f l f l}$ skin showed no significant difference with that of $\mathrm{Col} 5 \mathrm{a} 2^{f / f}$ littermate controls (Figure 2H), indicating lack of the hyperextensibility characteristic of cEDS, ${ }^{12}$ and of the $\mathrm{Col} 5 \mathrm{al}{ }^{+/-}$cEDS mouse model, ${ }^{14}$ and even of constitutively $\mathrm{ColS}^{+/-}$heterozygous null adult mice. ${ }^{16}$

\section{Ultrastructural Analysis Reveals Ubc-Cre; Col5a $2^{f l f l}$ Mice to Have cEDS-Like Dermal Collagen Fibrillar Aggregates}

cEDS is characterized, in part, by large-diameter aggregates of collagen fibrils with cross-sectional cauliflower-like contours. ${ }^{12}$ However, as null COL5A2 alleles have yet to be identified in cEDS patients, ${ }^{12}$ and as we previously found $\mathrm{Col}_{5} \mathrm{a}^{+/-}$mice to have dermal collagen fibrils with relatively mild abnormalities in contour and diameter, ${ }^{16}$ we sought to determine the extent to which $\mathrm{Ubc}$-Cre; $\mathrm{Col} 5 \mathrm{a} 2^{\text {fllf }}$ mice would have changes in dermal collagen fibril size and geometry. To this end, transmission electron microscopy was used to examine skin ultrastructure. $\mathrm{Ubc}-\mathrm{Cre}$; Col5a $2^{f / f l}$ and $C o l 5 a 2^{f / f l}$ mice were both injected with tamoxifen and sacrificed at 15 weeks of age, at which time subscapular skin was collected. Analysis using transmission electron microscopy demonstrated that $\mathrm{Ubc}$-Cre; Colsa ${ }^{f l f l}$ skin had readily apparent large-diameter collagen fibril aggregates with cross-sectional cauliflower-like contours (Figure 3B), highly similar to the similar fibril aggregates that are a cardinal feature in the skin of cEDS patients. ${ }^{16}$ This feature was absent from control Col5a $2^{f / f l}$ skin (Figure 3A). Examination of longitudinal collagen fibril profiles further emphasized the overall fibril disorganization and larger fibril diameters of $\mathrm{Ubc}-\mathrm{Cre}$; Colsa $2^{f / f l}$ skin (Figure 3, C and D). Quantitative analyses of fibril cross sections also showed Ubc-Cre; Col5a $2^{f / f l}$ skin to have a lower density of fibrils, compared to Col5a $2^{f l f l}$ controls $(P=0.027)$ (Figure 3E), likely due, at least in part, to the presence of fibril aggregates and larger-diameter fibril diameters, such that there are fewer fibrils per unit space. Indeed, quantitative examination showed $\mathrm{Ubc}$-Cre; $\mathrm{Col} 5 \mathrm{a} 2^{f / f l}$ skin to have a rightward shift in the distribution of collagen fibril diameters, with an extended right tail of atypically large-diameter sizes, ranging from 240 to $480 \mathrm{~nm}$. The data thus indicate both an overall increase in collagen fibril diameters as well as a subpopulation of fibril aggregates of abnormally large diameters (Figure 3F).

\section{Ubc-Cre; Col5a2 $2^{f l f l}$ Mice Have Impaired Wound Healing}

Because cEDS patients have delayed and aberrant wound healing, ${ }^{19}$ we used a wound-healing assay to determine whether $\mathrm{Ubc}$-Cre; $\mathrm{Col} 5 \mathrm{a} 2^{f l / f t}$ mice might also have deficits in wound healing. Toward this end, 5-mm-diameter full skin thickness punch biopsy specimens were excised from $U b c$ Cre; Colsa2 $2^{f / f l}$ and Col5a $2^{f / f l}$ control littermate mice. Wounds were sutured open to prevent contraction and to allow healing primarily by reepithelialization, extracellular matrix (ECM) deposition, and granulation tissue formation, the predominant mechanisms of wound healing in humans. Wounds were measured every other day for 2 weeks until the mice were sacrificed. Examination of wounds at 14 days revealed significantly delayed healing of $\mathrm{Ubc}$-Cre; $\mathrm{Col} 5 \mathrm{a} 2^{f / f l}$ wounds, for which healing was always incomplete, as evidenced by readily apparent nonconjoined wound edges (Figure 4A), whereas wounds of $\mathrm{Col} 5 \mathrm{a} \mathrm{2}^{f / \mathrm{fl}}$ controls reproducibly showed complete or nearly complete healing (Figure 4A). By 14 days after wounding, Ubc-Cre; Colsa $2^{f / f t}$ wounds remained approximately $60 \%$ open, whereas $\operatorname{Col} 5 a 2^{f / f t}$ wounds were essentially closed (Figure 4, B and C).

\section{Col5a2 Ablation Predisposes to Increased Abdominal Aortic Aneurysm Diameters and to Aortic Arch Dissection and Rupture in Mouse Aortic Aneurysm Models}

It has been reported that patients with cEDS have a higher incidence of dilated aortic roots. ${ }^{25}$ Therefore, the tensile strength of aortas of heterozygous Col5a1 $1^{+/-14}$ and $\mathrm{Col} \mathrm{a2}^{+/-16}$ mice were previously examined ex vivo and were shown to have decreased aortic tensile strength and stiffness. Thus, we previously suggested that deficits in col(V) content might predispose to aortic aneurysms. ${ }^{16}$ To test this, however, $\mathrm{Ubc}$-Cre; Col5a $2^{f / f t}$ mice, which had relatively small body mass (Figure 2, A and B), 


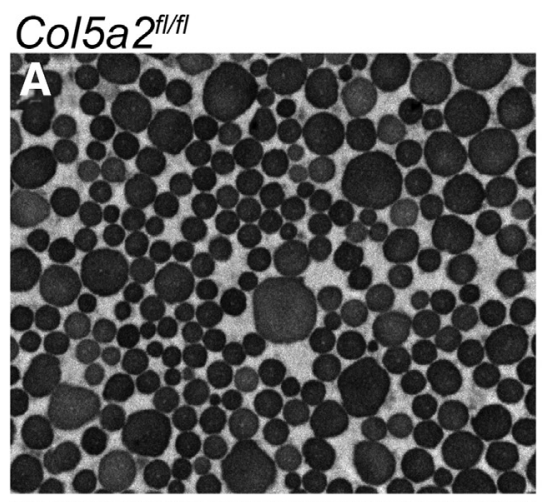

\section{Ubc-Cre; $\mathrm{Co} / 5 \mathrm{a} 2^{\mathrm{fl} / \mathrm{t}}$}
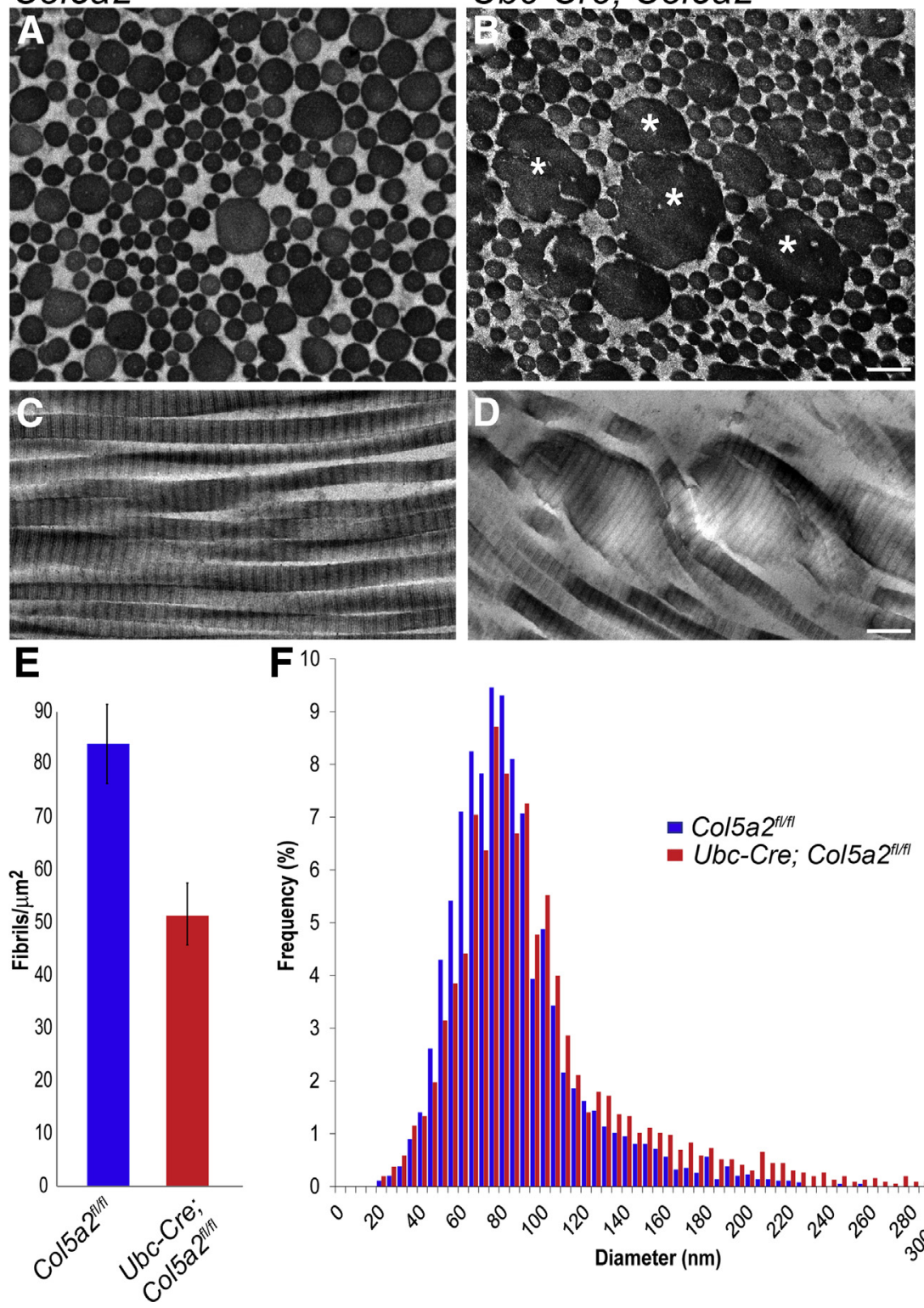

- Ubc-Cre; Col5a2 $2^{\text {il/tit }}$
Figure 3 Transmission electron microscopic analysis of skin from 15 -week-old Col5a $2^{f l f l}$ and Ubc-Cre; Col5a $2^{f l f l}$ mice. Ubc-Cre; Col5a2 ${ }^{f / f l}$ mice (B) have large aggregates of collagen fibrils, known as collagen cauliflowers ${ }^{12}$ (asterisks), with cross-sectional cauliflower-like profiles not seen in Col5a2 $2^{f l f l}$ controls (A). This alteration in fibril structure is highlighted in Ubc-Cre; Col5a $2^{f l f l}$ (D) and $\mathrm{Col}_{5 a 2^{f l / f l}}$ (C) longitudinal sections. Mutant mice (red) also have decreased fibril density (E) and a corresponding distribution skewed toward larger fibril sizes (F) compared with controls (blue). Data are presented as means \pm SEM (E). Scale bar $=200 \mathrm{~nm}(\mathbf{A}-\mathbf{D})$. also had large decreases in the sizes of their aortas, which were, therefore, not appropriate for comparison to controls in aorta induction experiments. It was also anticipated that $\mathrm{Ubc}$-Cre; $\mathrm{Col} 5 \mathrm{a} 2^{f / f t}$ mice might have poor survivability in such studies, because of a general failure to thrive. Thus, instead, Col5a $2^{f / f l}$ mice were crossed with transgenic Myh11-CreERT2 mice ${ }^{21}$ to produce Myh11-Cre; Col5a $2^{\text {flf }}$ mice, in which tamoxifen induction of Cre activity induces Col5a2 excision only in gastrointestinal smooth muscle cells (SMCs) and in vascular SMCs, ${ }^{26}$ with the latter a major source of secreted collagens in vessel walls. The Myh11-Cre transgene is Y linked, and all male littermates in these crosses contain the Myh11-Cre transgene. Some of these mice were treated with tamoxifen, for Col5a2 excision, whereas control littermates, with the same genotype, were treated with vehicle. Subsequent to tamoxifen/vehicle treatment, there were no differences in weight, nor were other gross external differences noted between the two groups of mice (data not shown). In a model in which elastase perfusion of the abdominal aorta induces AAAs, ${ }^{27}$ AAAs were induced in both groups of mice approximately 8 weeks after tamoxifen/ vehicle treatment. Interestingly, tamoxifen-treated mice had larger aneurysms than the vehicle-treated mice $(158.8 \%$ change $\pm 16.66 \%$ versus $110.0 \%$ change $\pm 10.75 \%$; $P<0.033$ ), indicating that Col5a2 ablation in aortic SMCs was sufficient to render the aorta more prone to dilation (Figure 5, $\mathrm{A}$ and $\mathrm{B})$.

The fact that SMCs represent only a fraction of cells of which the aortic wall is composed complicated our ability to 

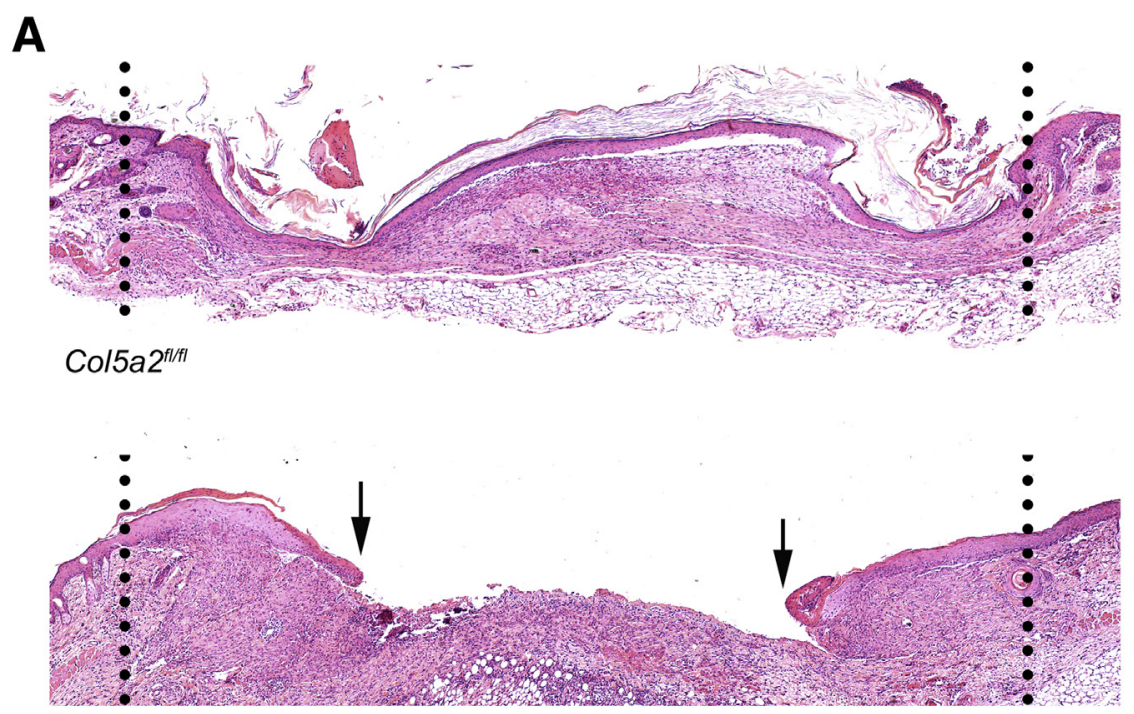

Ubc-Cre; Col5a2 $2^{f / t}$

B

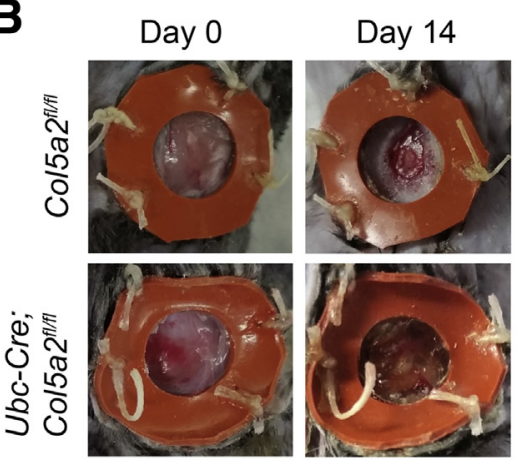

C

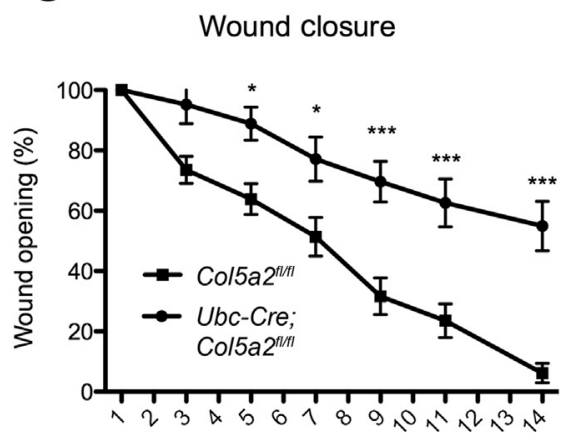

After wounding (days)
Figure 4 Delayed wound healing because of Col5a2 ablation. A: Representative images are shown of hematoxylin and eosin-stained histologic sections of Col5a2 $2^{f / f l}$ control and Ubc-Cre; Col5a $2^{f l f l}$ wounds at 14 days after wounding. Dotted lines denote wound margins; arrows denote edge of migrating epithelial tongues in a Ubc-Cre; Col5a $2^{f l f l}$ wound, whereas a Col5a2 $2^{f / f l}$ control wound is completely reepithelialized. B: Representative images at wounding and 14 days after injury. C: Quantification of open wound areas shows wound closure to occur significantly more

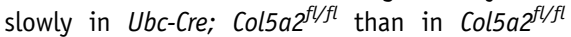
control wounds. Data are expressed as means \pm SEM (C). $n=9$ (C, Ubc-Cre; Col5a2 ${ }^{f l f l}$ wounds); $n=10$ (C, Col5a2 ${ }^{f l f l}$ wounds). ${ }^{*} P<0.05$, $* * * P<0.001$. ascertain the efficiency with which Col5a2 sequences were excised using the Myh11-Cre transgene (described above). In addition, although Myh11-Cre should excise Col5a2 sequences from mature SMCs, it would not excise Col5a2 sequences from progenitor/stem cells involved in SMC turnover and replacement, as such SMC-lineage cells do not yet express Myh11. ${ }^{28}$ Thus, SMCs from which Col5a2 had been excised could be replaced by progenitors that retained ability to express Col5a2. For these reasons, we thought that the Myh11-Cre; Col5a2 ${ }^{f l f l}$ studies described above might underrepresent $\mathrm{Col} 5 \mathrm{a} 2$ roles in aortic aneurysm pathogenesis. Thus, we sought to bolster such experiments by examining aortic aneurysm formation in Colsa $2^{+/-}$heterozygotes, in which all aortic cells would be Col5a2 haploinsufficient. For this study, Col5a2 $2^{+/-}$mice were crossed onto an $\mathrm{Ldlr}^{-1-}$ background, for use in the model system in which Ang II is infused into dyslipidemic mice to induce AAAs with various pathological features of human AAAs. ${ }^{29,30}$

In the Ang II infusion model, Col5 $\mathrm{a}^{+/-}$mice had marked increases in the incidence (Figure 6A), diameters (Figure 6B), and severity of vascular pathology (Figure 6C) of induced AAAs, compared with Col5a $2^{+l+}$ controls. Representative images of Col5 $a 2^{+/-}$and Col5a $2^{+/+}$Ang II-induced AAAs are shown in Figure 6D. Surprisingly, in addition to effects of Ang II infusion on AAA size, 6 of the $13 \mathrm{Col} \mathrm{a} 2^{+/-}$mice subjected to Ang II infusion died within the first 7 days of the start of Ang II treatment (Figure 7, A and B) of aortic arch rupture and dissection (Figure 7, C and D).

\section{Discussion}

The known molecular bases of cEDS include heterozygosity for COL5A1 null alleles or for missense COL5A2 mutations. $^{12}$ However, there have been no described cEDS patients with COL5A2 null alleles. In a previous study of the phenotypic consequences of null alleles for $\alpha 2(\mathrm{~V})$-encoding genes, we demonstrated ${ }^{16}$ that mice heterozygous for Col5a 2 constitutively null alleles had cEDS-related skin features that included increased extensibility and decreased tensile strength at high stress. However, a frank cEDS phenotype was lacking, as skin lacked hyperextensibility at low stress and large-diameter cauliflower-shaped aggregates of collagen fibrils, both of which are pathognomonic for 

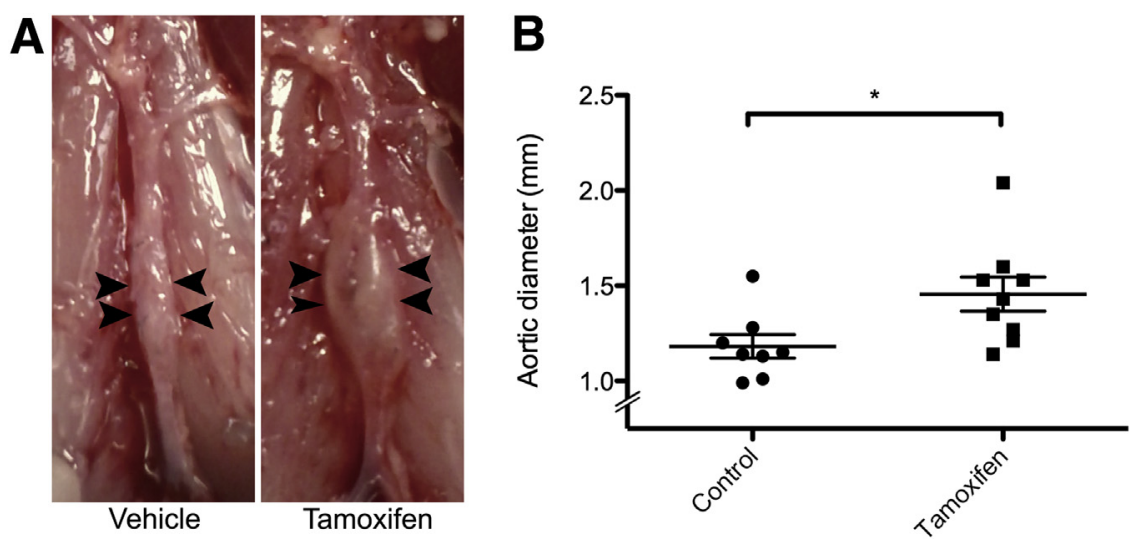

Figure 5 Col5a2 knockdown in vascular SMCs increases the diameters of elastase-induced AAAs. A: Image of representative AAAs, 2 weeks after elastase treatment, of Myh11-Cre; Col5a2 $2^{f / f l}$ mice treated with tamoxifen or with vehicle. Arrowheads indicate borders of aortic dilation. B: Quantification of AAA maximal external infrarenal aortic diameters. $n=9$ (A, tamoxifen treatment); $n=8$ (A, vehicle treatment). ${ }^{*} P<0.05$.
cEDS. We were interested in whether reducing Col5a2 expression to levels lower than those found in haploinsufficiency might produce a phenotype more typical of cEDS. Because complete loss of Col5a 2 function in mice is embryonic lethal, ${ }^{16}$ the present study used the first described conditional knockdown for Col5a2. Ubiquitous ablation at weaning allowed assessment of $\alpha 2(\mathrm{~V})$ chain roles in late perinatal development, in growth/maturation and in mature tissues.

Initial gross histologic examination of $\mathrm{Ubc}-\mathrm{Cre}$; $\mathrm{Col} 5 \mathrm{a} 2^{\mathrm{fl} / \mathrm{Al}}$ (Col5a2 knockdown) skin showed it to be extremely thin, with a compact, collagen-dense dermis in which dermal WAT was reduced to levels that were not readily detectable. This finding, along with our finding that abdominal fat depots were also much reduced in the Col5a 2 knockdown mice, even when normalized to body weight, suggests an important role for the $\alpha 2(\mathrm{~V})$ chain in WAT development and/or maintenance, rather than a skin-specific effect [although the effect of $\alpha 2(\mathrm{~V})$ loss was most dramatic in skin].

Interestingly, the extreme thinness of $\operatorname{Col} 5 \alpha 2$ knockdown dermis and lack of dermal WAT are not characteristic of cEDS. In contrast, transmission electron microscopy analysis showed Col5a2 knockdown skin to have the largediameter cauliflower-contoured collagen fibril aggregates pathognomonic of cEDS. Thus, precipitous postnatal loss of $\alpha 2(\mathrm{~V})$ chain expression produced a phenotype overlapping, but not identical with, cEDS.

Col5a2 knockdown skin had a $49 \%$ loss of breaking energy (toughness), compared with controls, similar to the extent of reduction of tensile strength in $\mathrm{ColSal}^{+/-}$skin in a cEDS mouse model. ${ }^{14}$ However, Col5a 2 knockdown skin differs from cEDS skin, and even from the skin of $\mathrm{Col}_{5} \mathrm{a}^{+/-}$heterozygotes, ${ }^{16}$ in lacking hyperextensibility, even under high stress. Thus, the presence of large

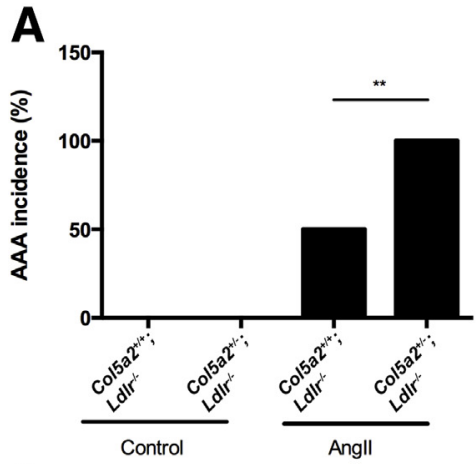

C

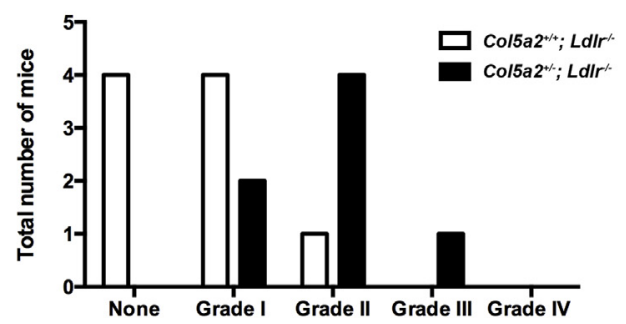

B

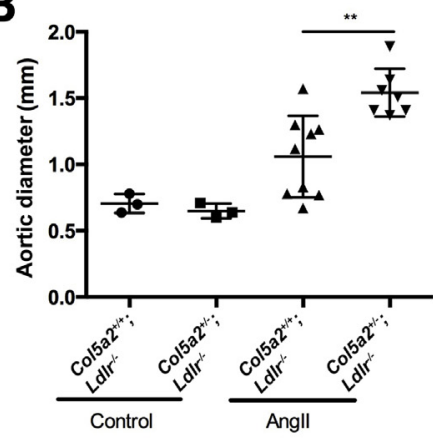

D

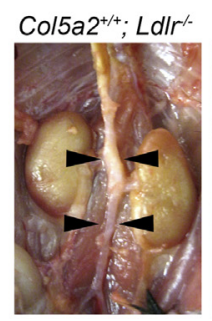

Figure 6 Col5a2 deficiency also exacerbates Ang II-induced AAA formation in mice. A: AAA incidence. B: Quantification of AAA maximal external suprarenal aortic diameter on day 28. An AAA is defined as percentage increase in aortic diameter of $\geq 50 \%$ (red dashed line). C: $A A A$ classification in Col5a2 $2^{+/+}$versus Col5a2 ${ }^{+/-}$mice after 28 days of Ang II infusion. D: Representative images of aortas. Arrowheads demarcate the area of the abdominal aorta treated with elastase. For comparing AAA incidence, Fisher's exact probability test was used. For comparison of aortic diameter differences, Mann-Whitney $U$ test was used. $n=3$ (A, both genotypes of saline controls); $n=9$ (A and C, Col5a2 ${ }^{+/+}$Ang II group); $n=7$ (A and C, Col5a2 ${ }^{+/-}$Ang II group). ${ }^{* *} P<0.01$. 


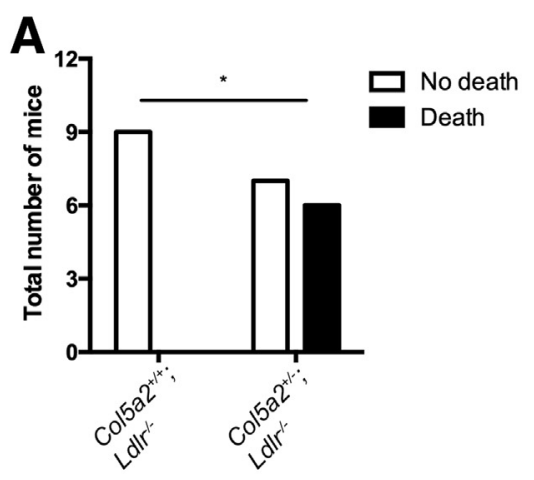

C

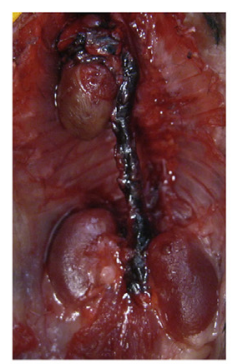

B

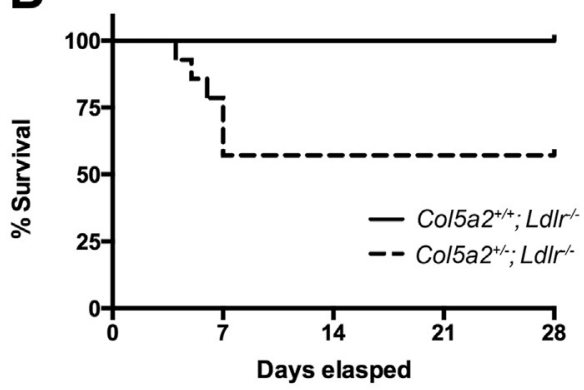

D

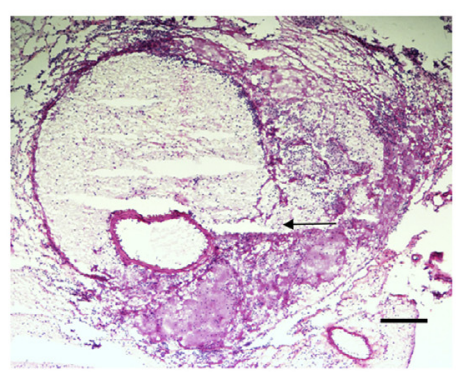

Figure 7 Col5a2 deficiency predisposes mice to aortic dissection and ruptures after Ang II infusion. A: Mortality due to aortic dissection and rupture within the first 7 days of Ang II treatment. Fisher's exact probability test was used to compare Col5a $2^{+/+}$versus $\mathrm{Col}_{52} 2^{+/-}$. Of the deaths, $50 \%$ were because of Debakey type I dissection, $16 \%$ were because of type II dissection, and $33 \%$ were because of type III dissection. B: Kaplan-Meier survival curve $(P=0.03$, log-rank test). C: Representative image of dissection. D: Hematoxylin and eosin-stained section of thoracic dissection and rupture (arrow). $n=9\left(\mathbf{A}, \mathrm{Col} 5 a 2^{+/+}\right)$; $n=13\left(\mathbf{A}\right.$, Col$\left._{5 a 2^{+/-}}\right) ; n=3$ (A, type I dissection); $n=1$ (A, type II dissection); $n=2$ (A, type III dissection). ${ }^{\star} P<0.05$. Scale bar $=200 \mu \mathrm{m}$ (D). cauliflower-shaped collagen fibril aggregates, but lack of hyperextensibility, in $\operatorname{Col} 5 \alpha 2$ knockdown skin unlinks these two cardinal cEDS features, and shows that the cEDS skin hyperextensibility does not rely on the presence of large cauliflower-shaped collagen fibril aggregates. We speculate that, although the cauliflower-shaped collagen fibril aggregates form in Col5 $\alpha 2$ knockdown skin because of $\alpha 2(\mathrm{~V})$ levels that, on tamoxifen-induced Col5a2 ablation, are lower than those found in haploinsufficiency, absence of hyperextensibility in Col5a2 knockdown skin derives from the persistence of some level of normal ECM deposited before tamoxifen treatment.

A seed-and-feed process has been proposed for the formation of fibrillar collagenous $\mathrm{ECM}^{31}{ }^{31}$ in at least some tissues. The seed phase of the process involves the extrusion of uniformly small-diameter collagen fibrils by stromal cells to form the fibrillar collagenous architecture of tissues in early prenatal development, whereas the feed phase occurs later in development and in homeostasis, and involves growth of collagen fibrils via fusion of small-diameter fibrils and accretion of collagen monomers onto fibril surfaces. Col(V) appears to play an important nucleation role in the seed phase of collagen fibrillogenesis, as collagen fibrils do not form in early embryonic tissues in the absence of $\operatorname{col}(\mathrm{V}) .{ }^{11}$ Our current findings, in which collagen cauliflower-shaped aggregates are caused by postnatal Col5a2 knockdown, indicate that aberrantly low levels of normal $\alpha 1(\mathrm{~V})_{2} \alpha 2(\mathrm{~V})$ heterotrimers during the feed phase are sufficient for formation of such aggregates. In contrast, the previous observation that constitutive Col5a2 haploinsufficiency results in hyperextensibility, ${ }^{16}$ together with the present observation that even lower levels of postnatal Col5a2 expression do not, suggests that insufficient $\alpha 1(\mathrm{~V})_{2} \alpha 2(\mathrm{~V})$ levels during the seed phase of fibrillar collagenous ECM may be primarily responsible for the skin hyperextensibility of cEDS.

As noted above, the extreme thinness of $\mathrm{Ubc}$-Cre; Col5 $\alpha 2^{f / f l}$ knockdown skin, deriving in large part from almost complete lack of dermal WAT, is not reminiscent of cEDS in humans, nor has it been reported for the Col5al $^{+/-}$cEDS mouse model. ${ }^{14}$ This difference in phenotypes may lie in the fact that decreased $\alpha 1(\mathrm{~V})$ chain levels result in only normal $\operatorname{col}(\mathrm{V})$, albeit at decreased levels, whereas decreased $\alpha 2(\mathrm{~V})$ chain levels result not only in lower levels of $\operatorname{col}(\mathrm{V})$, but also in the generation of an aberrant form of $\operatorname{col}(\mathrm{V})$. This is because, in the absence of sufficient $\alpha 1(\mathrm{~V})$ levels, excess $\alpha 2(\mathrm{~V})$ chains are unstable and are degraded, such that only normal $\alpha 1(\mathrm{~V})_{2} \alpha 2(\mathrm{~V})$ heterotrimers are deposited in tissues; however, in the absence of sufficient $\alpha 2(\mathrm{~V})$ chain levels, $\alpha 1(\mathrm{~V})$ chains can form stable $\alpha 1(\mathrm{~V})_{3}$ homotrimers. ${ }^{17,18,32}$ The result is that tissues in which $\alpha 2(\mathrm{~V})$ chains are at low levels would be affected not only by reduced deposition of $\alpha 1(\mathrm{~V})_{2} \alpha 2(\mathrm{~V})$ heterotrimers, but also by deposition of aberrant levels of $\alpha 1(\mathrm{~V})_{3}$ homotrimers. Thus, we speculate that aberrant ECM with lowered $\alpha 1(\mathrm{~V})_{2} \alpha 2(\mathrm{~V})$ content and elevated $\alpha 1(\mathrm{~V})_{3}$ content presents a microenvironment not conducive to adipogenesis and/or adipocyte maintenance. That the $\alpha 2(\mathrm{~V})$ chain may well play key roles in microenvironments that inform adipogenesis and/or adipocyte maintenance is supported by a study in which mice expressing a certain type of $\alpha 2(\mathrm{~V})$ chain with truncated $\mathrm{N}$-terminal non-triple helical 
sequences had dermal WAT up to sixfold thicker than wild type. ${ }^{33}$ Consistent with possible key roles for $\operatorname{col}(\mathrm{V})$ in WAT biology is the finding that adiponectin overexpression leads to reduced expression of $\operatorname{col}(\mathrm{V})$ genes, accompanying a suppression of fat accumulation, ${ }^{34}$ and our previous finding that knockout of the gene for the $\alpha 3(\mathrm{~V})$ chain of $\operatorname{col}(\mathrm{V})$ leads to subtle reductions in the thickness of dermal WAT and substantial changes to WAT function. ${ }^{6}$ Interestingly, the appearance of abnormally small miniadipocytes in the reduced mass of Col5a2 knockdown abdominal WAT depots is reminiscent of miniadipocytes reported in knockout mouse models in which ECM is rendered relatively rigid and not easily remodeled by extracellular proteases. ${ }^{35}$ Such ECM is thought to impair the hypertrophic response necessary to complete adipocytic maturation, ${ }^{35}$ suggesting that the Col5a2 knockdown ECM found in WAT may be similarly abnormally rigid and resistant to remodeling.

We demonstrate Ubc-Cre; Col5a $2^{f / f l}$ mice, like cEDS patients ${ }^{19}$ and Cols $\mathrm{al}^{+/-}$mice, ${ }^{15}$ to have deficits in wound healing. However, we speculate that, in the case of the Col5a2 knockdown mice, the paucity of dermal WAT may to some degree contribute to the severity in the deficits in wound healing, as both adipose-derived stem cells and mature adipocytes have been suggested to be important to the reepithelialization, revascularization, and fibroblast repopulation necessary to skin repair. ${ }^{36}$

As cEDS patients have a higher incidence of dilated aortic roots than normal ${ }^{25}$ and mice haploinsufficient for Colsal ${ }^{14}$ or $\mathrm{Col} 5 \mathrm{a} 2^{16}$ have aortas with diminished tensile strength, we sought to determine the extent to which lowered levels of $\alpha 2(\mathrm{~V})$ chains might affect the pathogenesis of aortic aneurysms. On treatment with tamoxifen, conditional Col5a2 knockdown in aortic smooth muscle cells was sufficient to increase the diameters of elastase-induced AAAs. Moreover, as tamoxifen has been shown to be protective against elastase-induced aneurysms in rodent models, in which it reduced aneurysm sizes by approximately $50 \%,{ }^{37}$ the effect of deficits in $\alpha 2(\mathrm{~V})$ levels on aneurysm progression may well have been underestimated in our study. However, although it seems unlikely, we cannot formally dismiss the possibility that tamoxifen may have had differential effects on $\mathrm{Ubc}$-Cre; Col5a $2^{f / f t}$ and $\mathrm{Col} 5 \mathrm{a} 2^{f / / f l}$ control mice.

In a follow-up study of $\mathrm{Col} 5 \mathrm{a} 2^{+/-}$heterozygotes, in which Ang II infusion induced AAAs with pathological features similar to those of human AAAs, ${ }^{29,30}$ not only did $\mathrm{Col}_{5 \mathrm{a}}{ }^{+/-}$mice have marked increases in the incidence, diameter, and severity of AAAs, compared with controls, but more than half died of aortic arch dissection and rupture. These data are exciting, in that they suggest that COL5A2 dysfunction in the general human population may predispose to aortic dilations, dissection, and rupture. That deficits in col(V) function can underlie loss of arterial integrity in humans is supported by recent reports of predisposition to rupture and dissection in the major arteries of a few cEDS patients with certain COL5Al missense mutations, ${ }^{38}$ the occurrence of an aortic aneurysm in a cEDS patient with a COL5A1 splice site mutation, ${ }^{39}$ and COL5A1 or COL5A2 gene variants of unknown significance in two nonsyndromic cases of aortic root aneurysms. ${ }^{39}$

In sum, a previous study of $\mathrm{Col} 5 \mathrm{a} 2^{+/-}$mice $^{16}$ together with the present study of conditional Col5a2 knockdown provide evidence indicating that insufficiency for $\alpha 2(\mathrm{~V})$ chains is unlikely to produce a frank cEDS-like phenotype, thus helping explain the absence of cEDS patients with COL5A2 null alleles. ${ }^{12}$ Moreover, the lack of an obvious phenotype in the absence of stress in $\mathrm{Col}_{5} \mathrm{a}^{+/-}$mice suggests that $C O L 5 A 2^{+/-}$individuals probably exist in the general human population, but would be unlikely, on routine clinical examination, to be diagnosed not only with cEDS, but perhaps with other clinical abnormalities as well. However, our findings that insufficiency for $\alpha 2(\mathrm{~V})$ chains can exacerbate AAA formation and predispose to dissecting and ruptured aneurysms of the aortic arch suggest COL5A2 as an important and novel candidate marker/target for the identification and treatment of nonsyndromic individuals prone to aortic aneurysm and dissection.

\section{References}

1. Fichard A, Kleman JP, Ruggiero F: Another look at collagen V and XI molecules. Matrix Biol 1995, 14:515-531

2. Niyibizi C, Fietzek PP, van der Rest M: Human placenta type V collagens: evidence for the existence of an alpha 1(V) alpha 2(V) alpha 3(V) collagen molecule. J Biol Chem 1984, 259:14170-14174

3. Rhodes RK, Miller EJ: Physicochemical characterization and molecular organization of the collagen A and B chains. Biochemistry 1978, $17: 3442-3448$

4. Kumamoto CA, Fessler JH: Biosynthesis of A, B procollagen. Proc Natl Acad Sci U S A 1980, 77:6434-6438

5. Dart ML, Jankowska-Gan E, Huang G, Roenneburg DA, Keller MR, Torrealba JR, Rhoads A, Kim B, Bobadilla JL, Haynes LD, Wilkes DS, Burlingham WJ, Greenspan DS: Interleukin-17-dependent autoimmunity to collagen type V in atherosclerosis. Circ Res 2010 , 107:1106-1116

6. Huang G, Ge G, Wang D, Gopalakrishnan B, Butz DH, Colman RJ, Nagy A, Greenspan DS: Alpha3(V) collagen is critical for glucose homeostasis in mice due to effects in pancreatic islets and peripheral tissues. J Clin Invest 2011, 121:769-783

7. Modesti A, Kalebic T, Scarpa S, Togo S, Grotendorst G, Liotta LA, Triche TJ: Type V collagen in human amnion is a $12 \mathrm{~nm}$ fibrillar component of the pericellular interstitium. Eur J Cell Biol 1984, 35:246-255

8. Gordon MK, Foley JW, Birk DE, Fitch JM, Linsenmayer TF: Type V collagen and Bowman's membrane: quantitation of mRNA in corneal epithelium and stroma. J Biol Chem 1994, 269:24959-24966

9. Birk DE, Fitch JM, Babiarz JP, Linsenmayer TF: Collagen type I and type $\mathrm{V}$ are present in the same fibril in the avian corneal stroma. J Cell Biol 1988, 106:999-1008

10. Birk DE, Fitch JM, Babiarz JP, Doane KJ, Linsenmayer TF: Collagen fibrillogenesis in vitro: interaction of types I and V collagen regulates fibril diameter. J Cell Sci 1990, 95(Pt 4):649-657

11. Wenstrup RJ, Florer JB, Brunskill EW, Bell SM, Chervoneva I, Birk DE: Type V collagen controls the initiation of collagen fibril assembly. J Biol Chem 2004, 279:53331-53337

12. Symoens S, Syx D, Malfait F, Callewaert B, De Backer J, Vanakker O, Coucke P, De Paepe A: Comprehensive molecular analysis demonstrates 
type V collagen mutations in over $90 \%$ of patients with classic EDS and allows to refine diagnostic criteria. Hum Mutat 2012, 33:1485-1493

13. Toriello HV, Glover TW, Takahara K, Byers PH, Miller DE, Higgins JV, Greenspan DS: A translocation interrupts the COL5A1 gene in a patient with Ehlers-Danlos syndrome and hypomelanosis of Ito. Nat Genet 1996, 13:361-365

14. Wenstrup RJ, Florer JB, Davidson JM, Phillips CL, Pfeiffer BJ, Menezes DW, Chervoneva I, Birk DE: Murine model of the EhlersDanlos syndrome: col5a1 haploinsufficiency disrupts collagen fibril assembly at multiple stages. J Biol Chem 2006, 281:12888-12895

15. DeNigris J, Yao Q, Birk EK, Birk DE: Altered dermal fibroblast behavior in a collagen $\mathrm{V}$ haploinsufficient murine model of classic Ehlers-Danlos syndrome. Connect Tissue Res 2016, 57:1-9

16. Park AC, Phillips CL, Pfeiffer FM, Roenneburg DA, Kernien JF, Adams SM, Davidson JM, Birk DE, Greenspan DS: Homozygosity and heterozygosity for null Col5a2 alleles produce embryonic lethality and a novel classic Ehlers-Danlos syndrome-related phenotype. Am J Pathol 2015, 185:2000-2011

17. Bentz H, Bachinger HP, Glanville R, Kuhn K: Physical evidence for the assembly of A and B chains of human placental collagen in a single triple helix. Eur J Biochem 1978, 92:563-567

18. Haralson MA, Mitchell WM, Rhodes RK, Kresina TF, Gay R, Miller EJ: Chinese hamster lung cells synthesize and confine to the cellular domain a collagen composed solely of B chains. Proc Natl Acad Sci U S A 1980, 77:5206-5210

19. Malfait F, Wenstrup RJ, De Paepe A: Clinical and genetic aspects of Ehlers-Danlos syndrome, classic type. Genet Med 2010, 12:597-605

20. Ruzankina Y, Pinzon-Guzman C, Asare A, Ong T, Pontano L, Cotsarelis G, Zediak VP, Velez M, Bhandoola A, Brown EJ: Deletion of the developmentally essential gene ATR in adult mice leads to agerelated phenotypes and stem cell loss. Cell Stem Cell 2007, 1:113-126

21. Wirth A, Benyo Z, Lukasova M, Leutgeb B, Wettschureck N, Gorbey S, Orsy P, Horvath B, Maser-Gluth C, Greiner E, Lemmer B, Schutz G, Gutkind JS, Offermanns S: G12-G13-LARG-mediated signaling in vascular smooth muscle is required for salt-induced hypertension. Nat Med 2008, 14:64-68

22. Committee for the Update of the Guide for the Care and Use of Laboratory Animals; National Research Council: Guide for the Care and Use of Laboratory Animals: Eighth Edition. Washington, DC, National Academies Press, 2011

23. Dunn L, Prosser HC, Tan JT, Vanags LZ, Ng MK, Bursill CA: Murine model of wound healing. J Vis Exp 2013, 75:e50265

24. Daugherty A, Manning MW, Cassis LA: Antagonism of AT2 receptors augments angiotensin II-induced abdominal aortic aneurysms and atherosclerosis. Br J Pharmacol 2001, 134:865-870

25. Wenstrup RJ, Meyer RA, Lyle JS, Hoechstetter L, Rose PS, Levy HP, Francomano CA: Prevalence of aortic root dilation in the EhlersDanlos syndrome. Genet Med 2002, 4:112-117
26. Thompson RW, Liao S, Curci JA: Vascular smooth muscle cell apoptosis in abdominal aortic aneurysms. Coron Artery Dis 1997, 8:623-631

27. Tsui JC: Experimental models of abdominal aortic aneurysms. Open Cardiovasc Med J 2010, 4:221-230

28. Psaltis PJ, Simari RD: Vascular wall progenitor cells in health and disease. Circ Res 2015, 116:1392-1412

29. Rush C, Nyara M, Moxon JV, Trollope A, Cullen B, Golledge J: Whole genome expression analysis within the angiotensin IIapolipoprotein E deficient mouse model of abdominal aortic aneurysm. BMC Genomics 2009, 10:298

30. Rateri DL, Howatt DA, Moorleghen JJ, Charnigo R, Cassis LA, Daugherty A: Prolonged infusion of angiotensin II in apoE(-/-) mice promotes macrophage recruitment with continued expansion of abdominal aortic aneurysm. Am J Pathol 2011, 179:1542-1548

31. Canty EG, Lu Y, Meadows RS, Shaw MK, Holmes DF, Kadler KE: Coalignment of plasma membrane channels and protrusions (fibripositors) specifies the parallelism of tendon. J Cell Biol 2004, 165:553-563

32. Imamura Y, Steiglitz BM, Greenspan DS: Bone morphogenetic protein-1 processes the NH2-terminal propeptide, and a furin-like proprotein convertase processes the $\mathrm{COOH}$-terminal propeptide of pro-alpha1(V) collagen. J Biol Chem 1998, 273:27511-27517

33. Andrikopoulos K, Liu X, Keene DR, Jaenisch R, Ramirez F: Targeted mutation in the col5a2 gene reveals a regulatory role for type $\mathrm{V}$ collagen during matrix assembly. Nat Genet 1995, 9:31-36

34. Khan T, Muise ES, Iyengar P, Wang ZV, Chandalia M, Abate N, Zhang BB, Bonaldo P, Chua S, Scherer PE: Metabolic dysregulation and adipose tissue fibrosis: role of collagen VI. Mol Cell Biol 2009, 29:1575-1591

35. Huang G, Greenspan DS: ECM roles in the function of metabolic tissues. Trends Endocrinol Metab 2012, 23:16-22

36. Shook B, Rivera Gonzalez G, Ebmeier S, Grisotti G, Zwick R, Horsley V: The role of adipocytes in tissue regeneration and stem cell niches. Annu Rev Cell Dev Biol 2016, 32:609-631

37. Grigoryants V, Hannawa KK, Pearce CG, Sinha I, Roelofs KJ, Ailawadi G, Deatrick KB, Woodrum DT, Cho BS, Henke PK, Stanley JC, Eagleton MJ, Upchurch GR: Tamoxifen up-regulates catalase production, inhibits vessel wall neutrophil infiltration, and attenuates development of experimental abdominal aortic aneurysms. J Vasc Surg 2005, 41:108-114

38. Monroe GR, Harakalova M, van der Crabben SN, Majoor-Krakauer D, Bertoli-Avella AM, Moll FL, Oranen BI, Dooijes D, Vink A, Knoers NV, Maugeri A, Pals G, Nijman IJ, van Haaften G, Baas AF: Familial Ehlers-Danlos syndrome with lethal arterial events caused by a mutation in COL5A1. Am J Med Genet A 2015, 167:1196-1203

39. Ziganshin BA, Bailey AE, Coons C, Dykas D, Charilaou P, Tanriverdi LH, Liu L, Tranquilli M, Bale AE, Elefteriades JA: Routine genetic testing for thoracic aortic aneurysm and dissection in a clinical setting. Ann Thorac Surg 2015, 100:1604-1611 